\title{
Measurable Categories and 2-Groups
}

\author{
Louis Crane and David N. Yetter \\ Department of Mathematics \\ Kansas State University \\ Manhattan, KS 66506
}

\begin{abstract}
Using the theory of measurable categories developped in Yet03, we provide a notion of representations of 2-groups more well-suited to physically and geometrically interesting examples than that using 2-VECT (cf. [KV94]). Using this theory we sketch a 2-categorical approach to the state-sum model for Lorentzian quantum gravity proposed in [CY03, and suggest state-integral constructions for 4-manifold invariants.
\end{abstract}

\section{Introduction}

In Section 2 we recall the relevant definitions and results from Yet03. In Section 3 we recall the relevant definitions and well-known results concerning 2-groups, discuss examples of particular importance for physics and group representation theory including the Poincaré 2-group first considered by Baez Bae02]. Section 4 develops the representation theory of 2-groups in the 2-category Meas. Section 7 outlines the common features of anticipated constructions both of a large family of new topological quantum field theories arising from the general theory and of a new model for quantum general relativity arising from the representation theory of the Poincaré 2-group.

\section{Measurable categories}

Throughout this work, we assume that all Borel spaces have measurable points. This restriction is necessary for the definition of "measurable functors" given below. However, it excludes only pathological examples: Euclidean space with either Borel or Lebesgue measure, the Borel structure on any locally compact group, and all discrete Borel spaces satisfy the condition.

We begin by recalling several definitions from Yet03.

Definition 1 A measurable field of Hilbert spaces $\mathcal{H}$ on a Borel space $(X, S)$ is a pair $\left(\mathcal{H}_{x}, \mathcal{M}_{\mathcal{H}}\right)$, where $\mathcal{H}_{x}$ is an $X$-indexed family of Hilbert spaces, and $\mathcal{M}_{\mathcal{H}}=\mathcal{M}$ is a linear subspace of $\prod_{x \in X} \mathcal{H}_{x}$ (the product as vector-spaces) satisfying

1. $\forall \xi \in \mathcal{M} x \mapsto\|\xi(x)\|_{x}$ is measurable

2. $\forall \eta \in \prod_{x \in X} \mathcal{H}_{x} x \mapsto\langle\eta(x) \mid \xi(x)\rangle_{x}$ is measurable for all $\xi \in \mathcal{M}$ implies $\eta \in \mathcal{M}$

3. $\exists\left\{\xi_{i}\right\}_{i=1}^{\infty} \subset \mathcal{M}$ such that $\xi_{i}(x)_{i=1}^{\infty}$ is dense in $\mathcal{H}_{x}$ for all $x \in X$

An almost measurable field of Hilbert spaces $\mathcal{H}$ on a Borel space $(X, S)$ is a pair $\left(\mathcal{H}_{x}, \mathcal{M}_{\mathcal{H}}\right)$ as above, satisfying 1. and 2., but not necessarily 3. 
Definition $2 A$ measurable field of bounded operators $\phi$ from $\mathcal{H}$ to $\mathcal{K}$, for $\mathcal{H}$ and $\mathcal{K}$ (almost) measurable fields of Hilbert spaces is an $X$-indexed family of bounded operators $\phi_{x} \in B\left(\mathcal{H}_{x}, \mathcal{K}_{x}\right)$ such that $\xi \in \mathcal{M}_{\mathcal{H}}$ implies $\phi(\xi) \in \mathcal{M}_{\mathcal{K}}$, where $\phi(\xi)_{x}=\phi_{x}\left(\xi_{x}\right)$.

A measurable field of bounded operators is bounded if $x \mapsto\left\|\phi_{x}\right\|_{x}$ is a bounded real-valued function (Here \|\|$_{x}$ denotes the operator norm on $B\left(\mathcal{H}_{x}, \mathcal{K}_{x}\right)$.)

We can then organize these into a category:

Definition 3 The category of measurable fields of Hilbert spaces on $(X, S)$ has as objects all measurable fields of Hilbert spaces on $(X, S)$ and as arrows all bounded fields of bounded operators on $X$. Source, target, identity arrow and composition are obvious. We denote this category by $\operatorname{Meas}(X, S)$.

Similarly, the category of almost measurable fields of Hilbert spaces on $(X, S)$ has as objects all almost measurable fields of Hilbert spaces on $(X, S)$ and as arrows all bounded fields of bounded operator between them. We denote this category by $\operatorname{AlMeas}(X, S)$.

As in Yet03 these categories in turn are organized into a 2-category.

In describing the representations of 2-groups we need only invertible functors, we do not at first need to consider the full theory of measurable functors developed in Yet03. In describing the representations it is a matter of indifference whether we work in the 2-category Meas of Yet03, with all measurable functors as 1-arrows, or the 2-category $\mathbf{M e a s}_{a d d}$, with $\mathbb{C}$-linear additive functors as arrows.

Recall from Yet03

Theorem 4 Any additive functor with an additive inverse $\Phi: \operatorname{Meas}(X, S) \rightarrow \operatorname{Meas}(Y, T)$ is equivalent to a functor induced by pullback along an invertible measurable transformation $\tilde{\Phi}: Y \rightarrow$ $X$.

and

Theorem 5 Any $\mathbb{C}$-linear natural endomorphism of an invertible additive functor is given by fiberwise scalar multiplication by an bounded scalar valued function. 2-dimensional composition is given by multiplication of functions, and 1-dimensional composition with 1-arrows is given by pullback.

In describing the intertwiners, however, it will be necessary to choose either Meas or Meas $\mathbf{a d d}_{\text {. }}$ We prefer the former, as its structure is better understood.

For any measurable field of Hilbert spaces $\mathcal{K}$ on $X \times Y$ and a $Y$-indexed family of measures on $X,\left\{\mu_{y}\right\}$, let

$$
\Phi_{\mathcal{K},\left\{\mu_{y}\right\}}(\mathcal{H})_{y}=\int_{X}^{\oplus} \mathcal{H}_{x} \otimes \mathcal{K}_{<x, y>} d \mu_{y}(x)
$$

with $\mathcal{M}_{\Phi(\mathcal{H})}$ given as the closure under condition 2 of the set $\left\{\int_{A_{y}}^{\oplus} \eta(x) \otimes \kappa(x) d \mu_{y}(x) \mid \eta \in \mathcal{M}_{\mathcal{H}} ; \kappa \in\right.$ $\left.\mathcal{M}_{\mathcal{K}}\right\}$.

Definition 6 A functor from $\operatorname{AlMeas}(X, S)$ to $\operatorname{AlMeas}(Y, T)$ is measurable if it $\mathbb{C}$-linear equivalent to one of the following form $\Phi_{\mathcal{K},\left\{\mu_{y}\right\}}$

$A$ measurable functor from $\operatorname{Meas}(X, S)$ to $\operatorname{AlMeas}(Y, T)$ is the restriction of a measurable functor from $\operatorname{AlMeas}(X, S)$ to $\operatorname{AlMeas}(Y, T)$, while a measurable functor from $\operatorname{Meas}(X, S)$ to $\operatorname{Meas}(Y, T)$ is the factorization of a measurable functor from $\operatorname{Meas}(X, S)$ to $\operatorname{AlMeas}(Y, T)$ through Meas $(Y, T)$, provided it admits such a factorization. 


\section{2-Groups}

Definition 7 A categorical group is a group object in the category of (small) categories.

It turns out that a categorical group is necessarily a groupoid: an amusing little exercise using the middle-four interchange law for the functoriality of the group law and the covariant(!) functor ${ }^{-1}$ shows that every arrow has an inverse.

A moment's consideration reveals that categorical groups are in fact strict monoidal categories equipped with a very strong type of two-sided dual. Because of this, we denote the identity object by $I$ as is customary in monoidal categories. We may thus use the usual trick to regard categorical groups as 2-categories with a single object (and their 'objects' as 1-arrows, 'arrows' as 2-arrows, the group law as 1-dimensional composition, and the composition as 2-dimensional composition). When we do this, we refer to the resulting 2-category as a 2-group.

It is also easy to see that the group law on any categorical group $\mathfrak{C}$ induces group structures on $\operatorname{Ob}(\mathfrak{C})$ and $\operatorname{Arr}(\mathfrak{C})$.

Categorical groups have been studied previously as models of homotopy 2-types (cf. BS76] Yet93). Brown and Spencer BS76] show that a categorical group is equivalent to a crossed module:

Definition $8 A$ crossed module is a homomorphism of groups $\partial: E \rightarrow G$ together with an action $\triangleright$ of $G$ on $E$ by automorphisms, such that

$$
\begin{gathered}
\partial(g \triangleright e)=g(\partial e) g^{-1} \\
(\partial e) \triangleright \varepsilon=e \varepsilon e^{-1}
\end{gathered}
$$

The group $G$ is called the base group, while the group $E$ is called the fiber group (other authors call $E$ "the principal group", but we prefer the name fiber group to emphasise a similarity with fiber bundles).

The equivalence given by Brown and Spencer [BS76] arises as follows: Given a categorical group $\mathfrak{G}$ we let $G=O b(\mathfrak{G})$, the group of arrows of $\mathfrak{G}$, and $E \subset \operatorname{Arr}(\mathfrak{G})$ be the group of all arrow with $I$, the identity object, as source. $\partial$ is then the restriction of the target map to $E$, while the action of $G$ on $E$ is given by conjugation (in the group of arrows) by the (identity arrow of) the object.

Conversely, given a crossed module, one can form a categorical group by taking $G$ as the group of objects, and the semidirect product of $E$ and $G$ with product $(e, g)(\varepsilon, \gamma)=(e g \triangleright \varepsilon, g \gamma)$ as group of arrows. Source and target are given by source $(e, g)=g$, target $(e, g)=g \partial(e)$; the identity arrow on $g \in G$ is $\left(1_{I}, g\right)$, and composition is given by $(e, g)(f, h)=(e \dot{f}, g)$ whenever $h=g \partial(e)$.

The reader is left to complete the proof or refered to BS76.

We will be particularly interested in categorical groups with a somewhat simpler structure:

Definition 9 A categorical group is automorphic if all of its arrows are automorphisms.

In the crossed module picture, this is equivalent to the map $\partial$ being the trivial homomorphism I. Also observe that in this case the fiber group is necessarily abelian: the categorical composition provides a group law for which the group law in the crossed module structure is given by homomorphism. It then follows by the theorem of Eckmann-Hilton [EH62] that the two group laws coincide and are abelian. We will apply the adjective "automorphic" to the structure whether we consider it as a categorical group, a crossed module, or a 2-group.

We can use group representations to construct automorphic categorical groups, including the example closely related to conjectural constructions for lorentzian quantum gravity, the Poincaré 2-group. 
Definition 10 Let $G$ be a group, and $V, \rho$ be a representation of $G$, the representational categorical group $\rho \downarrow G$ is the automorphic categorical group given as a crossed module with base group $G$ and fiber group $V,+$ by the action $g \triangleright v=\rho(g)(v)$.

In the case of $G=S O(3,1)$, with $\mathbb{R}^{4}, \rho$ the natural action of $S O(3,1)$ by rotations (and boosts) on Minkowski space, the representational categorical group is the Poincaré 2-group of [Bae02], which we denote $\mathfrak{P}$.

\section{The Representation Theory of 2-groups}

As with groups, the study of representations means the study of maps to particularly nice or well understood examples. Early attempts (cf. Barret and Mackaay [?]) to represent 2-groups in one or another of the versions of the category $2-V E C T$ first describe by Kapranov and Voevodsky KV94 have foundered on the paucity of examples: with the exception of categorical groups with (pro)finite groups of objects, there will be too few representations to provide a satisfying theory. Indeed, representations in $2-V E C T$ cannot be collectively faithful unless the group of objects is profinite.

The theory of measurable categories was developed in Yet03, motivated by the weak analogy to similar problems in the representation theory of non-compact groups, precisely to overcome this difficulty.

Definition $11 A$ (measurable) representation of a 2-group $\mathfrak{G}$ is a 2-functor $R: \mathfrak{G} \rightarrow$ Meas. $A$ 1-intertwiner between two representations $R$ and $R^{\prime}$ is a 2-natural transformation $\phi: R \rightarrow R^{\prime}$. A 2-intertwiner between two (parallel) 1-intertwiners $\phi: R \rightarrow R^{\prime}$ and $\psi: R \rightarrow R^{\prime}$ is a modification $m: \phi: \rightarrow \psi$.

As with representations of groups, the task of understanding the representations of a 2-group consists primarily in decomposing arbitrary representations into simpler ones, and understanding the irreducible or indecomposable examples. Before this can be attempted, however, it is necessary to unwind the previous definition to identify the structures involved in terms of more familiar group theoretic and representation theoretic notions.

In the present work, we confine ourselves to the consideration of automorphic 2-groups.

Now for any 2-group $\mathcal{G}$, a representation $R$ assigns to the unique object a category $R(*)=$ $\operatorname{Meas}\left(X_{R}, S_{R}\right)$ for some measureable space $\left(X_{R}, S_{R}\right)$, and to each 1-arrow $G$ an invertible measurable functor $R(G): \operatorname{Meas}\left(X_{R}, S_{R}\right) \rightarrow \operatorname{Meas}\left(X_{R}, S_{R}\right)$. Now, by a result of Yetter Yet03 any such functor is induced by an invertible measurable transformation $R_{G}:\left(X_{R}, S_{R}\right) \rightarrow\left(X_{R}, S_{R}\right)$. Thus at the level of 1-arrows, switching to the language of crossed modules, we may say that a representation of a 2-group is specified by a measurable action of the base group on a Borel space.

In the case of an automorphic 2-group, any 2-arrow has the same source and target, and is thus of the form $g: G \rightarrow G$. The image is thus a natural automorphism of the functor $R(G)$. 1-dimensional composition with $G^{-1}$ reduces the problem of describing these to describing the natural automorphisms of the identity functor on Meas $\left(X_{R}, S_{R}\right)$. Now by the result of Yet03] any natural transformation from the identity functor to itself is determined by an bounded scalar valued function. The preservation of 2-dimensional composition imposes the condition that, for $\eta: I \rightarrow I$, the values $R(\eta)(x)$ for each $x \in X$ form a character of the fiber group $E$, while preservation of the 1-dimensional composition of 2-arrows with 1-arrows imposes the condition that $R(F \circ g): R(F \circ G) \rightarrow R(F \circ G)$ (resp. $R(g \circ H): R(G \circ H) \rightarrow R(G \circ H)$ ) is the left (resp. right) translation of the function $R(g)(x)$ by $R(F)$ (resp. $R(H)$ ). 
To understand this better, recall that the 2-arrows of an automorphic 2-group form a group under the 1-dimensional composition $\circ$ which is isomorphic to the semidirect product of $E$ and $G$. We may thus index them by pairs $(\eta, G)$, where $\eta: I \rightarrow I$. In these terms, the 1- and 2- dimensional compositions are given by $(\eta, G) \circ(\epsilon, H)=(\eta G \triangleright \epsilon, G H)$ and $(\eta, G)\left(\eta^{\prime}, G\right)=\left(\eta \eta^{\prime}, G\right)$ respectively.

It is an amusing exercise to verify directly in terms of this formulation that the middle-four interchange law holds.

In terms of this indexing, what we denoted $R(\eta)(x)$ above is $R(\eta, I)(x)$.

The images of all the 2-arrows may be identified with bounded functions $R(\eta, G)(x)$ which multiply the fiber at $x$ before the action of $R(G)$ displaces it. With this identification, the preservation of the 1-dimensional composition becomes

$$
R(\eta, G)(x) R(\epsilon, H)(G(x))=R(\eta G \triangleright \epsilon, G H)(x)
$$

Observe that for any 1-arrow $G$, and $e$ the identity in the fiber group, we have $R(e, G)(x) \equiv 1$. This fact, together with the condition above, gives

$$
R(\eta, G)(x)=R(\eta, G)(x) R(e, H)(G(x))=R(\eta, G H)(x)
$$

and

$$
R(\epsilon, H)(G(x))=R(e, G)(x) R(\epsilon, H)(G(x))=R(G \triangleright \epsilon, G H)(x)
$$

From the first, we see that the bounded function representing 2-arrows $(\eta, G)$ is independent of $G$. Thus, we may let $\mathcal{R}(\eta)(x)=R(\eta, G)(x)$ for any (and thus all) $G$.

In terms of this, the second condition becomes

$$
\mathcal{R}(\epsilon)(G(x))=\mathcal{R}(G \triangleright \epsilon)(x)
$$

A simple calculation shows that this condition, together with the preservation of 2-dimensional composition implies the preservation of 1-dimensional composition.

We have thus obtained the following:

Proposition 12 A representation of an automorphic 2-group with base group $\mathcal{G}$ and fiber group $E$ is given by a measurable action of $\mathcal{G}$ on a measurable space $(X, S)$, together with an $X$-indexed family $\mathcal{R}(\epsilon)(x)$ of characters of $E$, which is $\mathcal{G}$-equivariant in the sense that

$$
\mathcal{R}(\epsilon)(G(x))=\mathcal{R}(G \triangleright \epsilon)(x)
$$

Note that if the action of the base group $G$ on the Borel space $X$ is transitive, then choosing a point of $x \in X$ gives an indexing of the representations by characters of the fiber group assigned to $x$. If the action base group is also free, the representations with the given action of the base group are indexing is by all the characters of the fiber group. Otherwise, the indexing is by the characters fixed by the stablizer of $x$.

This indexing, is, however, dependent on the necessarily non-canonical choice of a point in the $G$-equivariant Borel space $X$.

In the case of a non-transitive action, choosing a point in each orbit gives a indexing of the representations with the given action of the base group by maps from the orbits to the set of characters of the fiber group.

We now turn to the task of giving a similar description to the 1-intertwiners for representations of an automorphic 2-group. 
Fix two representations $R$ and $S$, and let $X$ and $Y$ respectively be the measure space to which each maps the unique object.

Now, a 1-intertwiner $\Phi$ is a 2-natural transformation. It thus assigns to the unique object $*$ a measurable functor $\Phi_{*}$. As we will only require a description up to natural equivalence, we may assume that $\Phi_{*}=\Phi_{\mathcal{K},\left\{d \mu_{y}\right\}}$ for some $\mathcal{K}$, a measurable field of Hilbert spaces on $X \times Y$, and $d \mu_{y}$ measures on $X$.

To each 1-arrow $G$ (element of the base group in the crossed module picture), it assigns a filler for the square

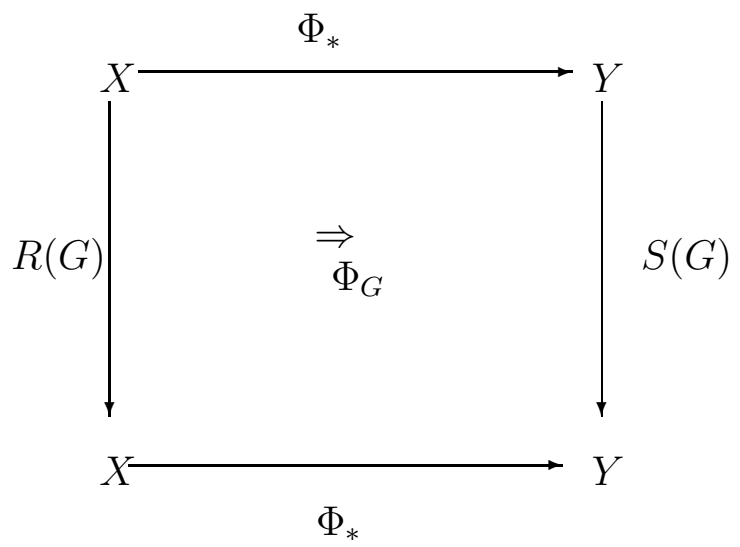

so that the composition of 1-arrows (the group law in the base group) is carried to pasting composition, and for each 2-arrow $\eta: G \rightarrow G$, the "pillow"

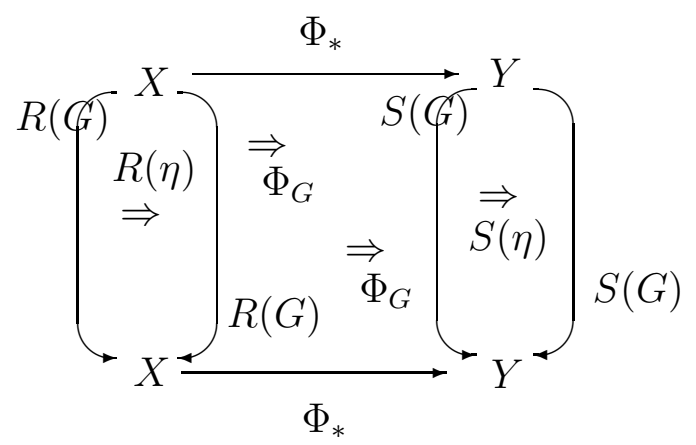

commutes.

As with the representations themselves, we must now unwind this definition to discover what more it entails in more familiar terms. Using the additivity properties shown in Yet03, it suffices to consider the components of the filler $\Phi$ at partial measurable line bundles. The action of the base group (1-arrows) provides a restriction on the partial measurable line bundles: they must be equivariant under the action, and thus supported on orbits.

The filler $\Phi_{G}$ thus has as components bounded fields of operators

$$
\Phi_{G, y}(\mathcal{H}): \int_{x \in X}^{\oplus} \mathcal{H}_{R(G)(x)} \otimes \mathcal{K}_{<x, y>} d \mu_{y}(x) \rightarrow \int_{x \in X}^{\oplus} \mathcal{H}_{x} \otimes \mathcal{K}_{<x, S(G)(y)>} d \mu_{S(G)(y)}(x)
$$

The passage of the composition of 1-arrows to pasting composition is then given by the condition

$$
\Phi_{G H, y}=\Phi_{G, y} \Phi_{H, S(G)(y)}
$$

The commutativity of the pillow for $\eta: G \rightarrow G$ becomes 


$$
\Phi_{G, y} \cdot \mathcal{S}(\eta)(S(G)(y))=\int^{\oplus} \cdot \mathcal{R}(\eta)(R(G)(x)) d \mu_{y}(x) \Phi_{G, y}
$$

but, since multiplication by a field of scalars commutes with any field of bounded operators, this becomes the condition that $\Phi_{G, y}$ coequalizes $\mathcal{S}(\eta)(S(G)(y))$ and $\int^{\oplus} \cdot \mathcal{R}(\eta)\left(R(G)(x) d \mu_{y}(x)\right.$, and thus, since the $\Phi_{G, y}$ is invertible that they are equal.

It therefore follows for all $\eta: G \rightarrow G$ and all $y \in Y$ that $\mathcal{R}(\eta)(R(G)(x))=\mathcal{S}(\eta)(S(G)(y))$ $\mu_{y}$-almost-everywhere.

However, the condition on the fields of characters which define the 2-arrow part of a representation allows us to replace this condition with $\mathcal{R}(G \triangleright \eta)(x)=\mathcal{S}(G \triangleright \eta)(x) \mu_{y}$-a.e. But, since this must hold for all $G$ and $\eta$, if we replace $\eta$ with $G^{-1} \triangleright \eta$, the condition reduces to $\mathcal{R}(\eta)(x)=\mathcal{S}(\eta)(y)$ $\mu_{y}$-a.e.

Consideration of the direct integral of the source, target, and fields of operators $\Phi_{G, y}$ with respect to any measure on $Y$ equivariant with respect to the action of the 1-arrows given by $S$ shows that any such direct integral carries a representation of the group of 2-arrows under 1-dimensional composition. But, it is a representation with additional structure: The base group acts by operators which are the composition of a diagonal operator with the translation operator on the underlying measure space defined by $S$. The fiber group acts by multiplication by the equivariant field of characters given by $S$. And, finally, the spaces on which the diagonal operators and characters act are themselves direct integrals with respect to some fibered measure of a measurable field of Hilbert spaces on $X$, the underlying Borel space of the source.

This last observation, together with the fact that the group of 2-arrows under 1-dimensional composition is the semidirect product of the fiber and base groups suggests that the representation theory of 2-groups may have applications to classical group representation theory in addition the the applications to topology and physics which motivated it.

Observe in the case of $\mathfrak{P}$ that, decomposing the representation into irreducible representations of the base group gives the same type of decomposition considered in the case of Dirac's expansors for $S O(3,1)$ in CY03. The connection with Dirac's expansors is even more intimate: an important family of irreducible representations of $\mathfrak{P}$ correspond to the orbits of $S O(3,1)$ acting on $\widehat{M^{4}}$ (which may be identified with Minkowski space), and are thus the energy levels for the system of harmonic oscillators used by Dirac. Thus the expansors are a quantization of the objects of our 2-category as conjectured in CY03.

Finally, we must consider how to describe the 2-intertwiners. A modification assigns to each object of a bicategory a 2-arrow between the 1-arrows assigned by the source and target, such that the pillows

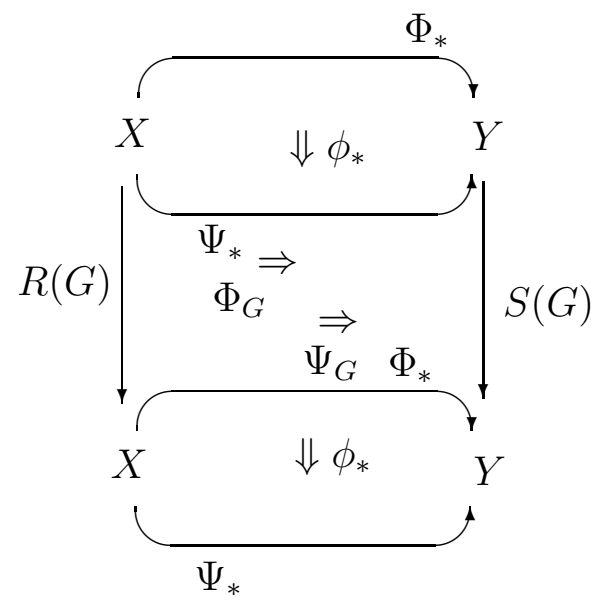


commute.

Thus in our case, we need a single 2-arrow. Using the same sort of reasoning as in the case of representations and 1-intertwiners to restrict our attention to the behavior on partial measurable line bundles, we can show the following: If $\Phi$ and $\Psi$ are 1-intertwiners with $\Phi_{*}=\Phi_{\mathcal{K},\left\{d \mu_{y}\right\}}$ and $\Psi_{*}=\Phi_{\mathcal{L},\left\{d \nu_{y}\right\}}$, a 2-intertwiner $\phi: \Phi \rightarrow \Psi$ is given by a bounded field of operators

$$
\phi_{y}: \int_{x \in X}^{\oplus} K_{<x, y>} d \mu_{y}(x) \rightarrow \int_{x \in X}^{\oplus} L_{<x, y>} d \nu_{y}(x)
$$

such that the pillows commute.

Again, for any measure on $Y$ equivariant under the base group, taking direct integrals gives a recognizable structure: the 2-intertwiner becomes an intertwiner in the ordinary sense between the representations of the base group (only) arising by taking direct integrals of the source and target 1-intertwiners. This intertwiner is, however, given by a diagonal operator with respect to the direct integral structure.

\section{Tensor Products}

As observed in Yet03 the bicategory Meas admits a monoidal structure induced by the cartesian product of measure spaces.

Definition 13 Let $\odot:$ Meas $\times$ Meas $\rightarrow$ Meas be given on objects by $(X, S) \odot(Y, T)=(X \times$ $Y, S * T)$, where $S * T$ denotes the Borel structure induced by all products $A \times B$ for $A \in S$ and $B \in T$.

$\odot$ is then defined on 1-arrows as follows: for $F=\Phi_{\mathcal{K}\left\{d \mu_{\xi}\right\}}$ and $G=\Phi_{\mathcal{L},\left\{d \nu_{\theta}\right\}} F \odot G: \operatorname{Meas}(X) \odot$ $\operatorname{Meas}(Y) \rightarrow \operatorname{Meas}\left(X^{\prime}\right) \odot \operatorname{Meas}\left(Y^{\prime}\right)$ is given on objects by

$$
\mathcal{H} \mapsto \int_{(x, y) \in X \times Y}^{\oplus} \mathcal{H}_{(x, y)} \otimes \mathcal{K}_{<x, \xi>} \otimes \mathcal{L}_{<y, \theta>} d \mu_{\xi}(x) \times d \nu_{\theta}(y)
$$

And by the same formula mutatis mutandis on arrows.

$\odot$ on 2-arrows $\phi: F \rightarrow F^{\prime}$ and $\psi: G \rightarrow G^{\prime}$, where $F=\Phi_{\mathcal{K}\left\{d \mu_{\xi}\right\}}, F^{\prime}=\Phi_{\mathcal{K}^{\prime}\left\{d \mu_{\xi}^{\prime}\right\}}, G=\Phi_{\mathcal{L},\left\{d \nu_{\theta}\right\}}$, and $G^{\prime}=\Phi_{\mathcal{L}^{\prime},\left\{d \nu_{\theta}\right\}}$, is given as follows:

From [Yet03] recall that a natural transformation $\phi$ between measurable functors $F$ and $F^{\prime}$ as above is determine by a measurable field of operators on $X^{\prime}$

$$
\left\{\phi_{\xi}: \int_{x \in X}^{\oplus} \mathcal{K} d \mu_{\xi}(x) \rightarrow \int_{x \in X}^{\oplus} \mathcal{K}^{\prime} d \mu_{\xi}^{\prime}(x)\right\}
$$

and similarly $\psi$ is given by a bounded field of operators $\left\{\psi_{\theta}\right\}$ on $Y^{\prime} . \phi \odot \psi$ is then the natural transformation obtained by pre- and post-composing $\left\{\phi_{x} \otimes \psi_{y}\right\}$ with the natural isomorphisms obtained by applying the distributivity of $\otimes$ over direct integrals and the categorical Fubini's Theorem of [Yet03]

Just as the tensor product structure on VECT induces a monoidal structure on categories of representations of groups, so the monoidal bicategory structure $\odot$ on Meas induces a monoidal bicategory structure on the bicategory of representations of any 2-group. 


\section{Additive Reflections as Projections}

It appears that the natural analogue at the level of 1-arrows for the projections occurring as labels of faces in the state-sum constructions in dimension 3 are additive reflections, in particular those induced by bimeasurable inclusions.

Recall that a reflection in the categorical sense is a pair of adjoint functors $i \vdash r$ such that the unit $\eta: r(i) \rightarrow I d_{\text {source }(i)}=I d_{\text {target }(r)}$ is an isomorphism. We call a reflection between additive categories, both of whose functors are additive functors an additive reflection.

Recall from Yet03, that a function between measurable spaces is bimeasurable if both direct and inverse image preserve measurable sets. A bimeasurable inclusion $i:(X, S) \rightarrow(Y, T)$ induces an additive reflection $i_{*}: \operatorname{Meas}(Y, T) \rightarrow \operatorname{Meas}(X, S) \vdash i^{*} \operatorname{Meas}(X, S) \rightarrow \operatorname{Meas}(Y, T)$.

Additive reflections of this form provide a kind of decomposition of tensor products of representations of any automorphic 2-group: Consider two representations $R$ and $R^{\prime}$ with underlying Borel spaces $(X, S)$ and $\left(X^{\prime}, S^{\prime}\right)$.

Now, consider the representation $R \odot R^{\prime}$. Its underlying Borel space is $\left(X \times X^{\prime}, S * S^{\prime}\right)$, and the base group $\mathfrak{G}_{0}$ acts by the diagnonal action, and the fiber group acts by the (tensor) product of the characters assigned to the coordinates on the fibers of measurable fields.

The action of $\mathfrak{G}_{0}$ decomposes $X \times X^{\prime}$ into orbits, each of which inherits a Borel structure from the product, and thus is included by a bimeasurable inclusion.

Now it is clear that representations in which the base group acts transitively on the underlying Borel space are irreducible in the sense of admitting no proper non-empty subobjects. (Note, the linear structure here exists at the level of 1- and 2-arrows, not objects, so the minimal subrepresention is not a "0-representation" but the one with empty underlying Borel space.)

Additive relections along inclusions of orbits thus provide a "decomposition" of any representation into irreducibles. Moreover, both functors in these additive reflections are measurable: each is represented by a measurable line bundle on the product concentrated on the graph of the inclusion and direct integration with respect to measures concentrated on the image (or preimage) of the point.

\section{Coloring triangulations}

Although it is beyond the scope of the present work to give complete constructions of either 4dimensional TQFT's or the model for quantum gravity which are the principal motivations for considering representations of 2-groups in Meas, we wish to give an indication of the conjectural constructions for which we have developped this theory.

Both proceed by "coloring" the simplexes of a triangulated 4-manifold (considered as a spacetime in the QG case) with objects, 1-arrows, and 2-arrows of $\operatorname{Rep}(\mathfrak{G})$ (for $\mathfrak{G}=\mathfrak{P}$ in the QG case), organizing the colorings into a suitable measure space, and taking the volume of the measure space.

Notice that the more familiar state-sum constructions for TQFT's, and the construction for Euclidean quantum gravity proposed by Barrett and Crane [BC97] can be phrased in these terms: the product of $6 \mathrm{j}$ - or $10 \mathrm{j}$-symbols and (quantum) dimensions (and their reciprocals) is defining a discrete measure on the space of colorings.

In the present case, the space of colorings will not admit a discrete measure with the appropriate properties.

At least two versions of the construction readily come to mind. Both at least formally lead to TQFT's when applied to any automorphic 2-group, and either could, in the case of the Poincaré 2group $\mathfrak{P}$, lead to a good quantization of general relativity provided a suitable measure, concentrated 
on colorings which embody a quantum analog of the geometric restriction that a face as a bivector is the wedge of any two of its edges as vectors, can be discovered.

In the first, edges are colored with those representations which have the base group with its natural Borel structure as underlying Borel space. These are indexed by equivariant families of characters, which indexing set inherits a Borel structure from the Borel structure used to define Plancherel measure on the dual of the (abelian) fiber group.

Face labels are then the additive reflections from one edge label to the tensor product of the other two, and are thus indexed by a family of orbits in the product $\mathfrak{G}_{0} \times \mathfrak{G}_{0}$ with the diagonal action, namely those orbits for which product of a character in the equivariant family giving one tensorand with a character in the family giving the other tensorand lies in the family giving the label on the remaining edge. As the family of all the orbits of the diagonal action is indexed by the base group, and thus has a natural Borel structure, the family indexing the admissible face labels inherits a Borel structure.

In the second, edge are colored with those representation which have as underlying Borel space an orbit of the dual group $\hat{E}$ of the fiber group $E$ under the action of the base group. These orbits inherit a Borel structure from that used in defining the Plancherel measure on $\hat{E}$. Two variations are possible, one in which all such representations are permitted, and another in which we require that only the "tautologous" representation, in which at each point the Hilbert space is 1-dimensional and has the fiber group acting by the character which names the point, be used. For physical applications, this latter seems most promising as the permitted colorings are precisely the mass shells.

\section{References}

[Bae02] J. Baez. Higher yang-mills theory. e-print, abstract and full-text links at www.arxiv.org/abs/hep-th/0206130, 2002.

[BC97] J.W. Barrett and L. Crane. Relativistic spin networks and quantum gravity. e-print, abstract and full-text links at www.arxiv.org/abs/gr-qc/9709028, 1997.

[BS76] R. Brown and C.B. Spencer. \}-groupoids, crossed modules and the classifying space of a topological group. Proc. Kon. Akad. v. Wet., 79:296-302, 1976.

[CY03] L. Crane and D.N. Yetter. A more sensitive lorentzian state-sum. e-print, abstract and full-text links at www.arxiv.org/abs/gr-qc/0301017, 2003.

[EH62] B. Eckmann and P.J. Hilton. Group-like structures in categories. Ann. of Math., 145:227-255, 1962.

[KV94] M. Kapranov and V. Voevodsky. 2-categories and zamolodchikov tetrahedra equations. In Proc. Symp. Pure Math. 56, Part 2, pages 177-260. American Mathematical Society, 1994.

[Yet93] D.N. Yetter. Tqfts from homotopy 2-types. J. Knot Theory and its Ramifications, 2(1):113-123, 1993.

[Yet03] D.N. Yetter. Measurable categories. (in preparation), 2003. 